\title{
Case Report: An unusual cause of cyst infection in adult polycystic kidney disease (ADPKD)
}

\author{
ASunavala, RSoman, PSavaj, KDavda
}

\section{Introduction}

Although many parts of India are endemic for melioidosis, it is still considered an uncommon infection, often unsuspected and under-diagnosed.

Cyst infection is a serious complication of adult polycystic kidney disease (ADPKD) accounting for $15 \%$ of hospitalization in these patients. Non-specific clinical manifestations, poor yield on urine and blood cultures and the limitations of conventional imaging techniques makes the diagnosis especially challenging.

\section{Case report}

A 43 year old diabetic with chronic kidney disease secondary to ADPKD underwent a renal transplant in 2012 and was on maintenance immune-suppressants including low dose steroid. He presented to us in June 2017 with high grade fever and severe right sided flank pain. Initial blood and urine cultures were negative. CT scan of the abdomen revealed hypodensities within cysts of the right native kidney with peri-nephric and peri-ureteric fat stranding involving the transplant kidney as well. FDG PET scan was performed for further confirmation of cyst infection which showed soft tissue thickening along the wall of few large cysts in the right native kidney with significant uptake. The patient underwent right nephrectomy. Intra operatively, infected cysts with necrosis were seen and Burkholderiapseudomallei was isolated on tissue culture. On further questioning, the patient revealed that he had traveled to his native village on the Konkan coast a few weeks prior to his illness. He responded well to intravenous ceftazidime and oral cotrimoxazole and was afebrile on discharge.

\section{Discussion}

Renal abscesses or pyelonephritis due to melioidosis is a well-documented entity. However, only one such case of cyst infection in a patient with ADPKD has been reported earlier. Thus despite compatible risk factors, melioidosis was not considered in this patient. In fact, the epidemiological history was sought only after identification of the pathogen.

Precise identification of the organism was crucial in this case as Burkholderiapseudomallei has an unusual susceptibility pattern and requires prolonged duration of treatment. Currently used empiric treatment is unlikely to be effective.

Division of Infectious Diseases, P.D. Hinduja National Hospital \& Medical Research Centre, Mumbai, India Address for correspondence: Dr Ayesha Sunavala, Division of Infectious Diseases, P.D. Hinduja National Hospital \& Medical Research Centre, Mumbai, India+919820233453 Email:_drayeshasunavala@gmail.com https://orcid.org/0000-0003-3912-7642 